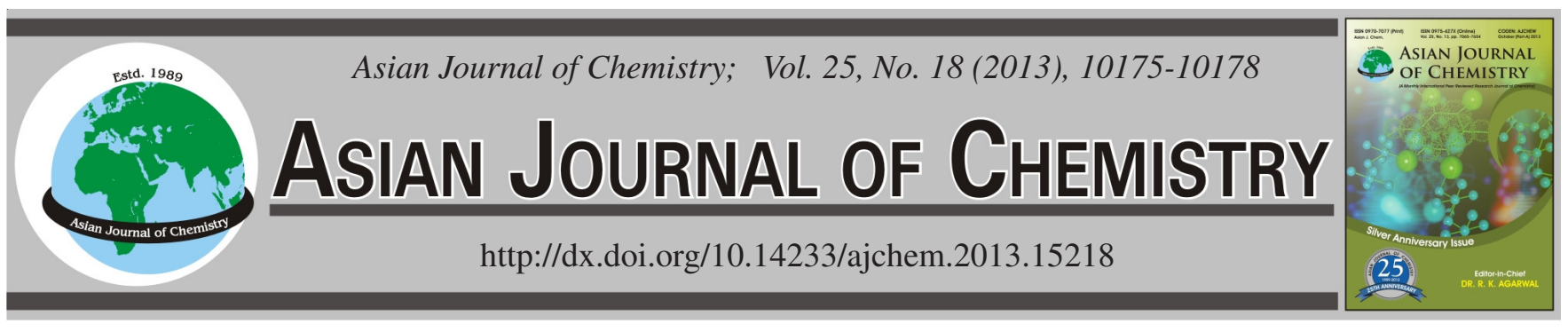

\title{
Acclimation of Anaerobic Culture Degrading 2,4-Dichlorophenol and 2,4,6-Trichlorophenol
}

\author{
T. CHENG ${ }^{1, *}$, C. $\mathrm{CHEN}^{2}$, T. PAN ${ }^{1}$ and W.F. XIE ${ }^{1}$
}

${ }^{1}$ Department of City Science, Jiangsu City Vocational College, Nanjing 210036, P.R. China

${ }^{2}$ School of Biology and Chemical Engineering, Jiangsu University of Science and Technology, Zhenjiang 212018, P.R. China

*Corresponding author: Fax: +86 25 86496516; Tel: +86 25 86496580; E-mail: wnchengting@ sina.com

(Received: 28 January 2013;

Accepted: 11 November 2013)

AJC-14360

\begin{abstract}
The experiment was conducted to examine the effect of acclimated culture on the 2,4-dichlorophenol and 2,4,6-trichlorophenol degradation. The variation of the chemical oxygen demand (COD) concentration in different degradation systems was also investigated. The results showed that, anaerobic culture began to degrade 2,4-dichlorophenol and 2,4,6-trichlorophenol almost without lag phase. In the early degradation period, the degradation rate of 2,4-dichlorophenol and 2,4,6-trichlorophenol was relatively lower, but the degradation rate improved obviously in the late degradation period. Compared to the fresh culture, the acclimated culture promoted the degradation rate of 2,4-dichlorophenol and 2,4,6-trichlorophenol. The degradation effect of the culture acclimated to 2,4-dichlorophenol and 2,4,6trichlorophenol was the best to the other culture. In addition, the degradation rate of organics was the highest in the system of $2,4-$ dichlorophenol and 2,4,6-trichlorophenol acclimated culture.
\end{abstract}

Key Words: Chlorophenols, 2,4-Dichlorophenol, 2,4,6-Trichlorophenol, Acclimation culture, Anaerobic biodegradation.

ᄂ - - - - - - - - - - - - - - - - - - - - - - - - - - -

\section{INTRODUCTION}

For many decades, chlorophenols (CPs) have been extensively used in the synthesis of many types of compounds such as pesticides, herbicides, dyes and wood preservers ${ }^{1-4}$. Some of the characteristics of chlorophenols, such as 2,4dichlorophenol and 2,4,6-trichlorophenol are their acute toxicity and poor biodegradability. Five chlorophenols are listed by the US Environmental Protection Agency (US EPA) as priority pollutants. Their discharge to the environment is of great concern ${ }^{5}$. Therefore, it has been necessary to develop an effective process for the destruction of such contaminants. Dai et $a l .{ }^{6}$ suggested that chlorophenols was extremely resistant to oxidative degradation, whereas it become easier in anaerobic conditions. Anaerobic biodegradation and biotransformation are important processes in determining the fate of pollutants in subsurface soil and sediments. Anaerobic biodegradation of chlorophenols has been reported in a variety of cultures inoculated with anaerobic digester sludge ${ }^{7}$, sediments $^{8}$, soils ${ }^{9}$ and ground water aquifers ${ }^{10}$. The investigations indicated that the inoculation microbe, acclimation substances and acclimation period could impact the degradability, degradation rate and degradation pathway of chlorophenols ${ }^{11,12}$. Boyd and Shelton ${ }^{11}$ investigated the anaerobic biodegradation of monochlorophenol and dichlorophenol isomers by unacclimated and acclimated sludge. The results demonstrated that the sludge acclimated to 4-chlorophenol (4-CP) could degrade 2-chlorophenol (2-CP), 3-chlorophenol (3-CP), 4-chlorophenol, 2,4dichlorophenol and 3,4-dichlorophenolthe sludge acclimated to 2-chlorophenol cross-acclimated to 4-chlorophenol could degrade 2,4-dichlorophenol, but did not utilize 3-chlorophenol. Cheok et al..$^{13}$ suggested that when the acidogenic sludge was acclimated with 2,4,6-trichlorophenol and developed 2,4,6trichlorophenol dechlorinating activity, pentachlorophenol (PCP) could be ortho-dechlorinated to 3,4,5-trichlorophenol.

In this study, we focused on the biodegradation of 2,4dichlorophenol and 2,4,6-trichlorophenol with fresh culture and acclimated culture in anaerobic conditions. The anaerobic culture was acclimated to individual 2,4-dichlorophenol, 2,4dichlorophenol and 2,4,6-trichlorophenol mixed pollutant, respectively. The degradation rate of organics in three degradation systems was also investigated.

\section{EXPERIMENTAL}

Microorganisms: A mixed anaerobic sludge used for this research was developed from a full-scale internal circulation reactor treating dye wastewater. The anaerobic sludge was first fed with $2.5 \mathrm{mg}$ of glucose per liter as the carbon source for 2 weeks, which enhanced the biological activity and COD removal rate was over $85 \%$ before the experiments.

Acclimation of anaerobic sludge: The acclimation of mixed culture was inoculated with the anaerobic sludge in 
2.5 L flask. The sludge was acclimated to 2,4-dichlorophenol, 2,4,6-trichlorophenol and 2,4-dichlorophenol, respectively. Each flask was fed glucose, chlorophenols stock solution and nutrients. Sodium bicarbonate was added to maintain a buffering capacity. Then the liquid was purged with nitrogen for 10 min to remove any residual dissolved oxygen completely. The flasks were sealed with rubber stoppers and incubated at $37^{\circ} \mathrm{C}$ for more than 2 months. During the acclimation period, the concentration of 2,4-dichlorophenol and 2,4,6trichlorophenol was increased gradually, nutrient and buffer solution was added to supply the nutrition for the microorganism. Meanwhile, a regular sample was taken from each flask for 2,4-dichlorophenol, 2,4,6-trichlorophenol and COD detection. The nutrient medium in flasks contained $\left(\mathrm{mg} \mathrm{L}^{-1}\right)$ : $\mathrm{KH}_{2} \mathrm{PO}_{4}$ (54), $\mathrm{K}_{2} \mathrm{HPO}_{4}$ (70), $\mathrm{NH}_{4} \mathrm{Cl}$ (106), $\mathrm{CaCl}_{2} \cdot 2 \mathrm{H}_{2} \mathrm{O}$ (15), $\mathrm{MgCl}_{2} \cdot 6 \mathrm{H}_{2} \mathrm{O}$ (20). The trace element solution contained (mg $\left.\mathrm{L}^{-1}\right): \mathrm{CoCl}_{2} \cdot 6 \mathrm{H}_{2} \mathrm{O}$ (500), $\mathrm{NiCl}_{2} \cdot 6 \mathrm{H}_{2} \mathrm{O}$ (50), $\mathrm{Na}_{2} \mathrm{SeO}_{3}(50)$, $\mathrm{CuCl}_{2} \cdot 2 \mathrm{H}_{2} \mathrm{O}$ (30), $\mathrm{ZnCl}_{2} 50, \mathrm{H}_{3} \mathrm{BO}_{3}$ (50), $\mathrm{MnCl}_{2} \cdot 4 \mathrm{H}_{2} \mathrm{O}$ (500), $\left(\mathrm{NH}_{4}\right)_{6} \mathrm{Mo}_{7} \mathrm{O}_{24} \cdot 2 \mathrm{H}_{2} \mathrm{O}$ (10). Additionally, the concentration of $\mathrm{NaHCO}_{3}$ buffer solution was $1,000 \mathrm{mg}$ per liter and the biomass concentration in each flask was approximately 4,200 mg per liter based on the volatile suspended solids (VSS) contents of anaerobic sludge.

Degradation of 2,4-dichlorophenol and 2,4,6-trichlorophenol by fresh and acclimated culture: Batch experiments were conducted using $250 \mathrm{~mL}$ serum bottles at $37^{\circ} \mathrm{C}$. The fresh (unacclimated) culture, the 2,4-dichlorophenol acclimated culture, the 2,4,6-trichlorophenol and 2,4-dichlorophenol mixed acclimated culture, was transferred to serum bottle with $50 \mathrm{~mL}$ nutrient medium and $2 \mathrm{~mL}$ trace element solution, respectively. Each bottle was spiked with 2,4-chlorophenol and 2,4,6-trichlorophenol stock solution to give an initial concentration of 20 and $10 \mathrm{mg} / \mathrm{L}$, respectively. $\mathrm{NaHCO}_{3}(1,000 \mathrm{mg} / \mathrm{L})$ was also added to maintain a buffering capacity. Bottles were then filled with deionized water and $\mathrm{pH}$ was adjusted with $\mathrm{HCl}$ and $\mathrm{NaOH}$ solution. The Initial $\mathrm{pH}$ was adjusted to 7 in all batch experiments. The liquid was purged with nitrogen for $10 \mathrm{~min}$ to remove any residual dissolved oxygen completely and then bottles were sealed with rubber stoppers and placed on a platform shaker and shaken continuously at 120 rpm over the course of experiments. The biomass concentration in each bottle was approximately $342 \mathrm{mg} / \mathrm{L}$ based on the volatile suspended solids (VSS) contents of anaerobic sludge. A regular sample was taken from each bottle for 2,4-dichlorophenol, 2,4,6-trichlorophenol and COD detection

Analytical methods: The sample was taken from bottle using a glass syringe. Analysis for 2,4-dichlorophenol and 2,4,6-trichlorophenol were performed using a Agilent LC-1260 HPLC system, equipped with a Lichrospher $\mathrm{C}_{18}$ inverse phase column. An L-2400 UV detector was used for the analysis and the detection wavelength was $290 \mathrm{~nm}$. The HPLC mobile phase was the mixture of purified water $(15 \%)$ and methanol $(85 \%)$ at flow rate of $1.0 \mathrm{~mL} \mathrm{~min}{ }^{-1}$. The injection volume was $10 \mu \mathrm{L}$ with an auto-sampler. Prior to HPLC analysis, sample solutions were filtered by $0.45 \mu \mathrm{m}$ membrane. Standard potassium dichromate method was used for the determination of chemical oxygen demand. The biomass was measured as volatile suspended solids using standard methods.

\section{RESULTS AND DISCUSSION}

Effect of fresh culture on the degradation of 2,4dichlorophenol and 2,4,6-trichlorophenol: The test was to investigate the biodegradation of the mixed pollutant for 2,4dichlorophenol and 2,4,6-trichlorophenol by the fresh culture (Fig. 1). Meanwhile, the biodegradation of individual 2,4dichlorophenol by the fresh culture was also investigated (Fig. 2). Fig. 1 showed the degradation of 2,4-dichlorophenol and 2,4,6-trichlorophenol by fresh culture. Fig. 1 indicated that the anaerobic culture had the potential of degrading chlorophenols and the anaerobic culture began to degrade 2,4-dichlorophenol and 2,4,6-trichlorophenol almost without lag phase. In the early degradation period $(0-120 \mathrm{~h})$, the anaerobic culture adapted to the toxicity of chlorophenols gradually and the degradation rate of 2,4-dichlorophenol and 2,4,6-trichlorophenol was only 32.25 and $32.21 \%$ in $120 \mathrm{~h}$, respectively. This suggested that the anaerobic culture demanded a comparatively long time to enrich a certain amount of the microorganism having the degradation activity for target contaminants.

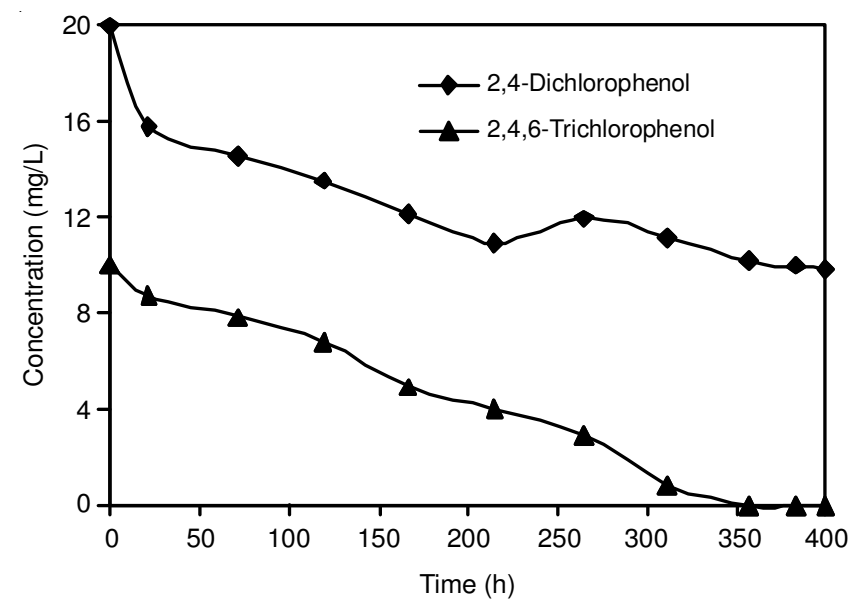

Fig. 1. Degradation of 2,4-dichlorophenol and 2,4,6-trichlorophenol by the fresh culture

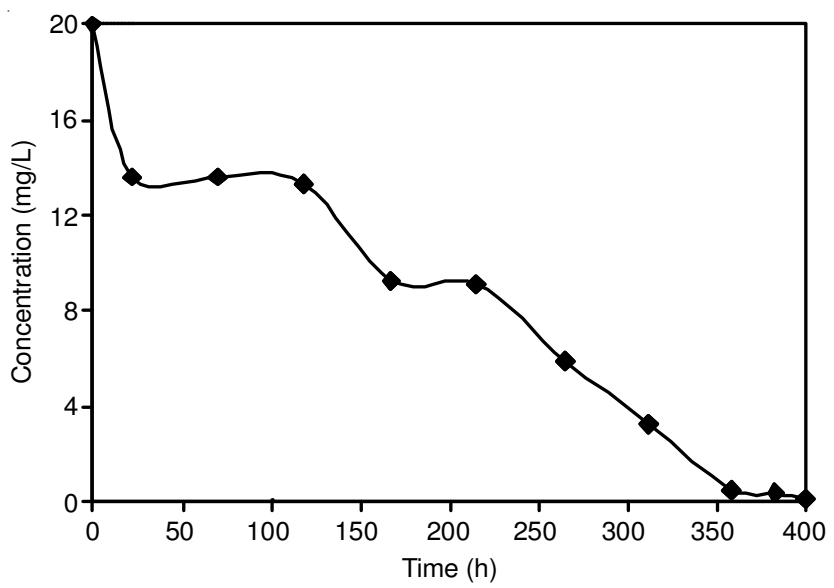

Fig. 2. Degradation of individual 2,4-dichlorophenol by the fresh culture

In addition, the degradation ability of fresh culture improved obviously with the adaptation of the target contaminants. When the fresh culture degraded 2,4-dichlorophenol and 2,4,6trichlorophenol in $310 \mathrm{~h}$, the degradation rate of 2,4dichlorophenol and 2,4,6-trichlorophenol was 42 and $91.49 \%$, 
respectively. The 2,4,6-trichlorophenol was degraded completely in $350 \mathrm{~h}$. The 2,4-dichlorophenol and 2,4,6-trichlorophenol degradation rate was 51 and $100 \%$ in $400 \mathrm{~h}$. It indicated that the degradation of 2,4,6-trichlorophenol was superior to the 2,4-dichlorophenol when the coexistence of 2,4-dichlorophenol and 2,4,6-trichlorophenol in anaerobic system.

Fig. 2 showed the individual 2,4-dichlorophenol degradation by the fresh culture. Fig. 2 indicated that the anaerobic culture began to degrade 2,4-dichlorophenol almost without lag phase. In the early degradation period (0-120 h), the degradation effect of individual 2,4-dichlorophenol was similar to the mixed pollutant of 2,4-dichlorophenol and 2,4,6-trichlorophenol (Fig. 1). The degradation rate of 2,4-dichlorophenol was $33.35 \%$ in $120 \mathrm{~h}$. Moreover, in the late degradation period, the degradation rate of 2,4-dichlorophenol was improved obviously. The degradation rate was 83.89 and $97.31 \%$ in 310 and $350 \mathrm{~h}$, respectively. The target pollutant (2,4-dichlorophenol) was degraded completely in $400 \mathrm{~h}$. It suggested the coexistence of 2,4,6-trichlorophenol and 2,4-dichlorophenol inhibited the degradation of 2,4-dichlorophenol by fresh culture.

Effect of 2,4-dichlorophenol acclimated culture on the degradation of 2,4-dichlorophenol and 2,4,6-trichlorophenol: Fig. 3 showed that the 2,4-dichlorophenol and 2,4,6-trichlorophenol degradation by the 2,4-dichlorophenol acclimated culture. Fig. 3 indicated that the degradation effect of 2,4dichlorophenol and 2,4,6-trichlorophenol mixed pollutant by the 2,4-dichlorophenol acclimated culture was superior to the fresh culture. In the degradation period of $120 \mathrm{~h}$, the degradation rate of 2,4-dichlorophenol and 2,4,6-trichlorophenol was only 29.78 and $30.17 \%$, respectively. However, in the late degradation period, the degradation ability of the 2,4dichlorophenol acclimated culture was improved obviously. After $260 \mathrm{~h}$, the 2,4,6-trichlorophenol was degraded completely. The 2,4-dichlorophenol degradation rate was 64.23 and $82.82 \%$ in 350 and $400 \mathrm{~h}$, respectively. That demonstrated the culture acclimated to 2,4-dichlorophenol accelerated the degradation of 2,4-dichlorophenol and 2,4,6-trichlorophenol.

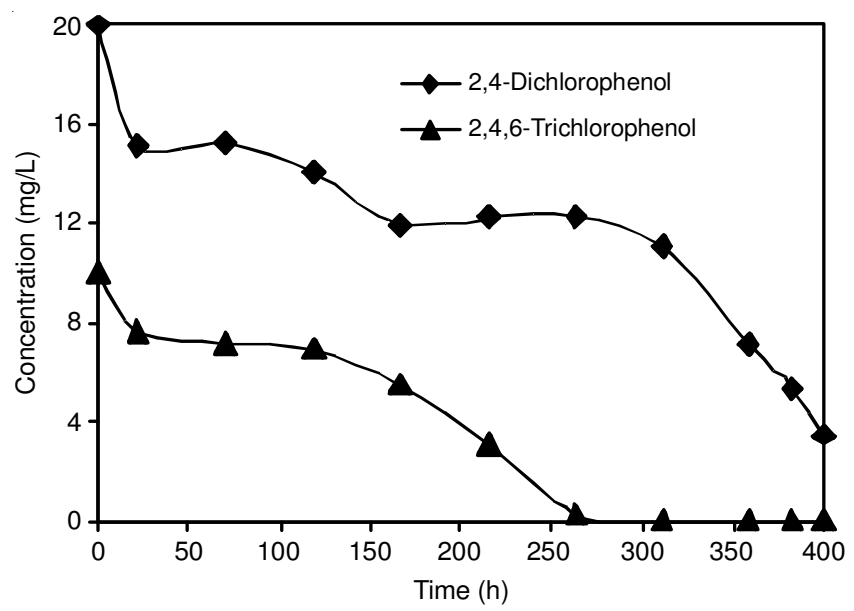

Fig. 3. Degradation of 2,4-dichlorophenol and 2,4,6-trichlorophenol by the 2,4-dichlorophenol acclimated culture

Effect of 2,4-dichlorophenol and 2,4,6-trichlorophenol mixed acclimated culture on the degradation of 2,4dichlorophenol and 2,4,6-trichlorophenol: Fig. 4 showed the degradation of 2,4-dichlorophenol and 2,4,6-trichlorophenol by the 2,4-dichlorophenol and 2,4,6-trichlorophenol mixed acclimated culture. Fig. 4 indicated that the degradation effect of 2,4-dichlorophenol and 2,4,6-trichlorophenol by the 2,4-dichlorophenol and 2,4,6-trichlorophenol mixed acclimated culture was the best to the other culture. After $120 \mathrm{~h}$, the degradation rate of 2,4-dichlorophenol and 2,4,6-trichlorophenol was 41.96 and $38.2 \%$, respectively. 2,4,6-Trichlorophenol was almost degraded completely in $210 \mathrm{~h}$ and the degradation rate of 2,4-dichlorophenol was $85.25 \%$. In addition, the 2,4-dichlorophenol degradation rate reached to $97.3 \%$ in $260 \mathrm{~h}$. The $2,4-$ dichlorophenol and 2,4,6-trichlorophenol was totally degraded in $310 \mathrm{~h}$. It indicated the culture acclimated to 2,4-dichlorophenol and 2,4,6-trichlorophenol promoted the degradation of 2,4-dichlorophenol and 2,4,6-trichlorophenol in anaerobic system. Moreover, the long-term coexistence of 2,4,6-trichlorophenol and 2,4-dichlorophenol was favour to the degradation of target contaminant. That indicated the culture acclimated to 2,4,6-trichlorophenol and 2,4-dichlorophenol presented higher potentiality for the degradation of 2,4-dichlorophenol and 2,4,6-trichlorophenol. Quan et al. ${ }^{14}$ suggested that, with the long-term coexistence of 4-chlorophenol and 2,4-dichlorophenol, the degradation rate of 2,4-dichlorophenol was higher than the situation of individual 2,4-dichlorophenol as acclimation substrate. The molecular structure of 2,4,6-trichlorophenol was similar to 2,4-dichlorophenol, that inferred the long-term coexistence of 2,4,6-trichlorophenol and 2,4-dichlorophenol induced more enzyme or enzyme system for the anaerobic culture degrading 2,4-dichlorophenol and 2,4,6-trichlorophenol and developed 2,4-dichlorophenol and 2,4,6-trichlorophenol dechlorinating activity, contaminants could be degraded.

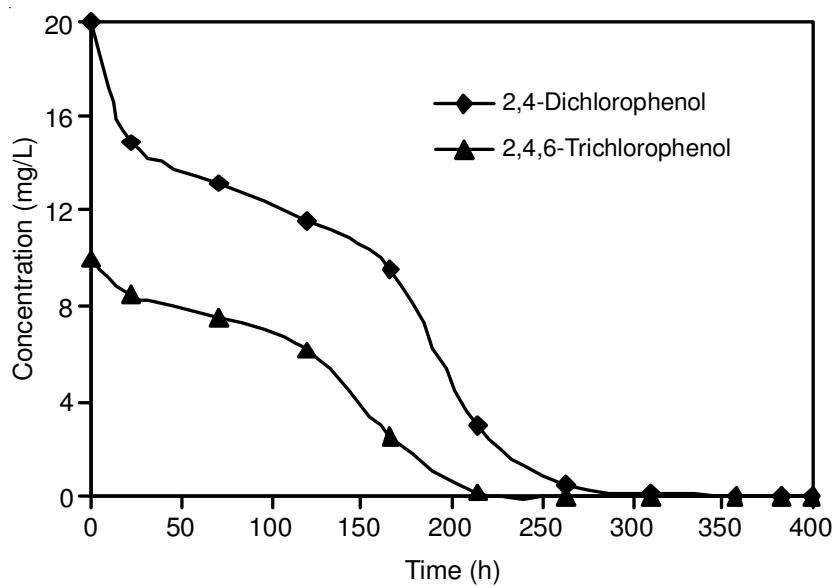

Fig. 4. Degradation of 2,4-dichlorophenol and 2,4,6-trichlorophenol by the 2,4-dichlorophenol and 2,4,6-trichlorophenol acclimated culture

Removal rate of COD in different anaerobic degradation systems: The variation of COD concentration in different degradation systems was measured, to reflect the anaerobic degradation of organics. Fig. 5 showed the removal rate of COD in different anaerobic degradation systems. Fig. 5 indicated that the removal rate of COD in different degradation systems had a relation to the degradation of 2,4-dichlorophenol and 2,4,6-trichlorophenol in some extent. The degradation of organics was very low in the system of fresh culture and the removal rate of COD was only $54 \%$. The removal rate of 


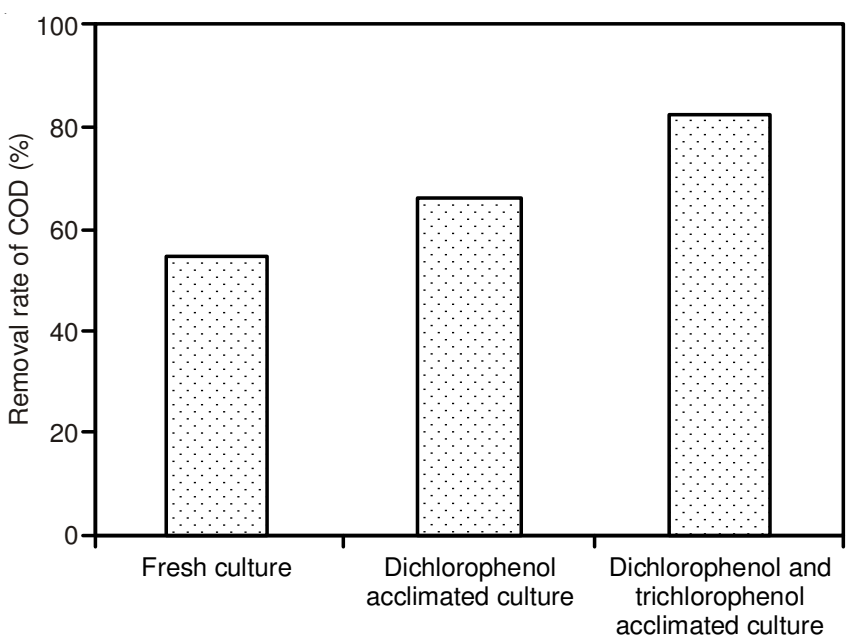

Fig. 5. COD removal rate in different degradation systems

COD was $66 \%$ in the system of 2,4-dichlorophenol acclimated culture. In addition, the degradation rate of organics was the highest in the system of 2,4-dichlorophenol and 2,4,6-trichlorophenol mixed acclimated culture, the removal rate of COD was more than $82 \%$.

\section{Conclusion}

Anaerobic culture had the potential of degrading 2,4dichlorophenol and 2,4,6-trichlorophenol and the culture began to degrade 2,4-dichlorophenol and 2,4,6-trichlorophenol almost without lag phase. In the early degradation period, the culture adapted to the toxicity of chlorophenols gradually and the degradation rate of 2,4-dichlorophenol and 2,4,6-trichlorophenol was relatively lower. However, the degradation rate of target contaminant improved obviously in the late degradation period. When the coexistence of 2,4-dichlorophenol and 2,4,6trichlorophenol in anaerobic system, the degradation of 2,4,6trichlorophenol was superior to the 2,4-dichlorophenol. Compared to the fresh culture, the acclimated culture improved the degradation effect of 2,4-dichlorophenol and 2,4,6-trichlo- rophenol obviously. The degradation effect of the culture acclimated to 2,4-dichlorophenol and 2,4,6-trichlorophenol was the best to the other culture. The long-term coexistence of 2,4,6-trichlorophenol and 2,4-dichlorophenol induced more enzyme or enzyme system for the culture degrading 2,4dichlorophenol and 2,4,6-trichlorophenol and thus in favour of the degradation of target contaminant. Moreover, the degradation rate of organics was the highest in the system of 2,4-dichlorophenol and 2,4,6-trichlorophenol acclimated culture, the removal rate of COD was $82 \%$. The COD removal rate in different degradation systems had a relation to the 2,4dichlorophenol and 2,4,6-trichlorophenol degradation in some extent.

\section{ACKNOWLEDGEMENTS}

The authors gratefully acknowledged the financial support of the "Twelfth Five-Year Plan" key task of Jiangsu City Vocational College (Grant No. 12SEW-Z-004).

\section{REFERENCES}

1. R. Valo, V. Kitunen and M.S. Salkinoja-Salonen, Water Sci. Technol., 17, 1384 (1985).

2. M. Czaplicka, Sci. Total Environ., 322, 21 (2004).

3. P. Juteau, R. Beaudet, G. McSween, F. Lupine, S. Milot and J.-G. Bisaillon, Appl. Microbiol. Biot., 44, 218 (1995).

4. M.D. Mikesell and S.A. Boyd, Appl. Environ. Microbiol., 52, 861 (1986).

5. L.H. Keith and W.A. Telliard, Environ. Sci. Technol., 13, 416 (1979).

6. Y.Z. Dai, H.C. Shi and J.P. Ji, Environ. Sci., 21, 40 (2000) (in Chinese).

7. R. Takeuchi, Y. Suwa, T. Yamagishi and Y. Yonezawa, Chemosphere, 41, 1457 (2000).

8. F.O. Bryant, D.D. Hale and J.E. Rogers, Appl. Environ. Microbiol., 57, 2293 (1991).

9. M.D. Mikesell and S.A. Boyd, Environ. Sci. Technol., 22, 1411 (1988).

10. J.M. Suflita and G.D. Miller, Environ. Toxicol. Chem., 4, 751(1985).

11. S.A. Boyd and D.R. Shelton, Appl. Environ. Microbiol., 47, 272 (1984).

12. D.S. Shen, X.Y. Xu and X.S. Feng, Acta Sci. Circumstant., 16, 299 (1996). in Chinese.

13. C.H. Mun, J. He and W.J. Ng, Water. Res., 42, 3789 (2008).

14. X.C. Quan, H.C. Shi and J.L. Wang, Acta Sci. Circumstant., 23, 69 (2003) (in Chinese). 